\title{
Frecuencia de Epidermophyton floccosum en dermatofitos aislados en un laboratorio de la Región de Valparaíso, Chile. Período 1980-2010
}

\author{
Rodrigo Cruz y Laura Carvajal
}

Universidad de Valparaíso, Valparaíso, Chile. Laboratorio de Micología (RC, LC). Hospital Carlos van Buren, Valparaíso, Chile. Unidad de Infectología (RC)

Los autores declaran no tener conflictos de interés.

Fuente de financiamiento: fondos propios del laboratorio de Micología de la Universidad de Valparaíso.

Recibido: 8 de mayo de 2017 Aceptado: 5 de marzo de 2018

Correspondencia a: Rodrigo Cruz Choappa rcruzchoappa@gmail.com

\author{
Frequency of Epidermophyton floccosum in isolated dermatophytea laboratory from \\ the Region of Valparaiso, Chile. Time frame: 1990-2010
}

Background: Dermatophytoses are frequent infections in human, which are produced by fungus genera Trichophyton, Microsporum and Epidermophyton. Aim: To determine frequency of E. floccosum in isolated dermatophyte in a laboratory from Valparaíso during the last three decades, in addition to knowing the most frequent localization, age and gender of patients affected by this agent. Method: All superficial culture reports, issued by the Universidad de Valparaiso's mycology laboratory in the last three decades, with E. floccosum, Microsporum spp and Trichophyton spp development, has been reviewed. Patients age, gender, location of the injury and culture report has been registered in an excel spreadsheet. Results: The total number of reports with dermatophyte development in the culture was 6,780. Only 26 cases show E. floccosum development, 73\% of this (19/26) were present on men with and average age of 37 years. Frequency did progressively fall since eighty's until 2000 decade. The age group most affected by this etiological agent was 30-36 years. The most frequently localization in both man and woman were foot plant and nails. Conclusions: Frequency of isolation did decrease progressively in dermatophytose by E. floccosum for the study period. Masculine gender and 36-60 age group were the most affected. The most frequently localization were foot plant and nails.

Key words: Epidermophyton floccosum; dermatophytose; frequency; Valparaiso.

Palabras clave: Epidermophyton floccosum; dermatofitosis; frecuencia; Valparaíso.

\section{Introducción}

L os dermatofitos son un grupo de hongos filamentosos estrechamente relacionados, capaces de invadir e infectar los tejidos queratinizados como piel, pelos y uñas, tanto del ser humano como de algunos animales, gracias a las queratinasas que poseen. Tres son los géneros que componen a este grupo de hongos: Trichophyton, Microsporum y Epidermophyton ${ }^{1,2}$. Según la procedencia de la queratina que utilizan se pueden clasificar en geofílicos o telúricos (Microsporum gypseum), en zoofílicos (Microsporum canis, Trichophyton mentagrophytes) y antropofílicos si se desarrollan en el ser humano (Trichophyton rubrum, Epidermophyton (loccosum) $)^{2}$.

El género Epidermophyton incluye sólo una especie patógena, E. floccosum, la que puede producir principalmente tiña de las ingles (eccema marginado de Hebra, tiña crural o cruris) y onicomicosis ${ }^{3}$. En los medios de cultivo se presenta con colonias visibles a los 7-9 días de incubación, son aterciopeladas, pulverulentas y de color amarillo-verdoso, las que se blanquean rápidamente y se vuelven flocosas. Microscópicamente se caracteriza por presentar abundantes macroconidios en racimo o aislados y por la ausencia de microconidios. Los macroconidios tienen forma de clavas, la pared es lisa y moderadamente gruesa, los extremos son redondeados y presentan de 1 a 9 septos $^{4}$.

Se han realizado estudios de las especies causantes de micosis superficiales en prácticamente todo el mundo, los que han mostrado que varían según las áreas geográficas, factores socioeconómicos, climáticos y migraciones ${ }^{4,5}$.

Se describen cambios significativos en la frecuencia y patrón clínico de las infecciones provocadas por algunas especies de dermatofitos en distintos países, incluido Chile $^{6-8}$. Varias publicaciones sobre dermatofitosis humanas en nuestro país han demostrado que las especies más frecuentes son T. rubrum, T. mentagrophytes y $M$. canis $^{9-14}$. En la Región de Valparaíso, T. rubrum es el agente más aislado en la mayoría de las lesiones del cuerpo y $M$. canis en tiña capitis; E. floccosum sólo representó entre 0,4 y 2,8\% del total de aislados en dos trabajos publicados ${ }^{9,10}$. No se han publicado artículos que muestren cambios de frecuencia o de presentación clínica de esta especie en las últimas décadas, en Chile ni en Latinoamérica. 
El objetivo de este trabajo fue determinar la frecuencia de E. floccosum en dermatofitos aislados en un laboratorio de la Región de Valparaíso durante las últimas tres décadas, conocer las localizaciones más frecuentes, los grupos etarios y sexo de los pacientes afectados por este agente.

\section{Materiales y Método}

Estudio descriptivo y retrospectivo. Se revisaron en libros de registro todos los cultivos de piel y anexos, y los informes de dermatofitosis con desarrollo de E. floccosum, Microsporum spp o Trichophyton spp emitidos por el Laboratorio de Micología de la Universidad de Valparaíso desde 1 de enero de 1980 hasta 31 de diciembre de 2010.

\section{Criterios de inclusión}

- Informes de cultivos con desarrollo de E. floccosum, Microsporum spp o Trichophyton spp durante las décadas de estudio. Sólo se incluyó un cultivo por paciente.

- Que se consignara sitio anatómico de la infección.

- Que el informe indicara la edad y sexo del paciente.

Diagnóstico micológico: Las muestras de las lesiones fueron obtenidas por personal entrenado y depositadas en placas de Petri estériles para evitar contaminación. El cultivo se realizó en agar Sabouraud glucosado y agar Lactrimel, ambos adicionados con cloranfenicol $(125 \mu \mathrm{g} /$

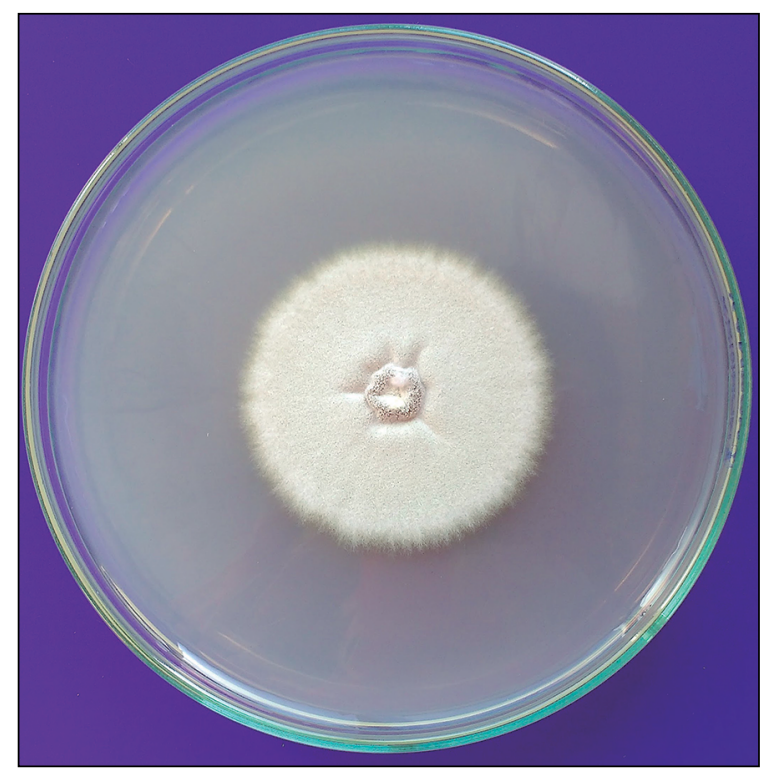

Figura 1. Se observa colonia de Epidermophyton floccosum a los 14 días de crecimiento. Obtenida de un paciente con tiña plantar. Laboratorio de Micología Universidad de Valparaíso. ml) e incubadas a 27 y $37^{\circ} \mathrm{C}$ durante dos semanas. La identificación de los hongos filamentosos fue en base a su morfo-fisiología (Figuras 1 y 2). Este método diagnóstico se mantuvo a través de las décadas estudiadas.

Se creó una planilla Excel donde se incorporaron las variables de edad, sexo, ubicación de la lesión y resultado del cultivo, para cada paciente. Los resultados se expresaron en números, porcentajes y tasas. Para analizar los cambios de frecuencias de infección por E. floccosum en el tiempo, los datos se agruparon en décadas de los ochenta, noventa y dos mil.

\section{Resultados}

El total de muestras obtenidas fue de 12.322 con el siguiente desglose: 1.558 en la década de los 80, 5.585 en la de los 90 y 5.179 en la del 2000.

El total de exámenes con informe de dermatofitosis fue de 8.475. Se descartaron 1.695 informes pues tenían presencia de artroconidios o micelio en el examen microscópico directo, pero sin desarrollo de algún dermatofito en el cultivo. En 6.780 hubo desarrollo de Epidermophyton, Trichophyton o Microsporum en los cultivos, 855 fueron en la década $80,3.128$ de los $90 \mathrm{y}$ 2.797 del 2000 (Tabla 1).

Sólo en 26 casos hubo desarrollo de E. floccosum; de éstos, 73\% (19/26) fueron hombres y con una edad promedio de 37 años.

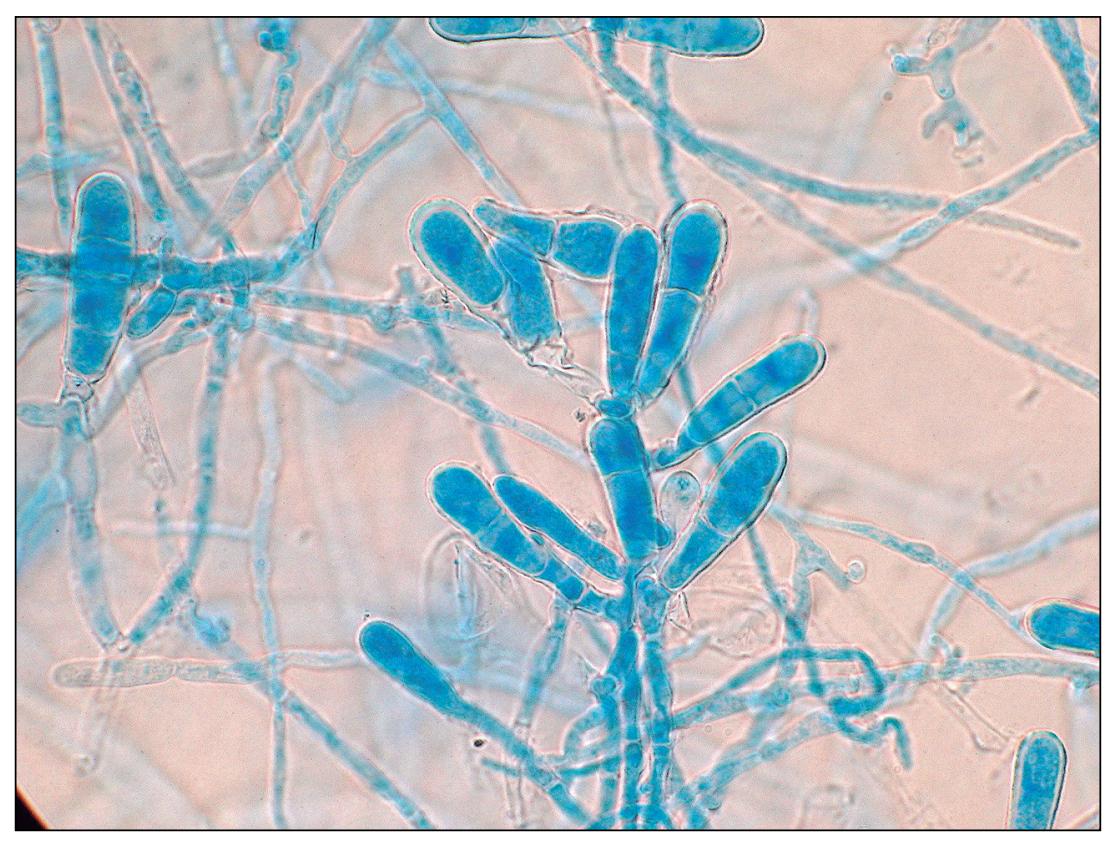

Figura 2. Se observan macroconidios en racimos, de paredes lisas, clavados, con uno o más septos, sin microconidios. 100 X. Obtenida de un paciente con tiña plantar. Laboratorio de Micología Universidad de Valparaíso. 


\begin{tabular}{|lccc|}
\hline \multicolumn{4}{|l|}{ Tabla 1. Distribución de dermatofitos agrupados por décadas } \\
\hline Género & Década 80 & Década 90 & Década 2000 \\
\hline Trichophyton spp & 738 & 2.813 & 2.629 \\
Microsporum spp & 108 & 304 & 162 \\
\hline Epidermophyton floccosum & 9 & 11 & 6 \\
\hline Total & 855 & 3.128 & 2.797 \\
\hline
\end{tabular}

Tabla 2. Distribución de aislamientos de E. floccosum según grupos etarios y sexo

\begin{tabular}{cccc}
\hline Grupo etario & Mujeres & Hombres & $\mathbf{n}$ de casos \\
$<8$ años & 0 & 0 & 0 \\
$8-15$ años & 1 & 1 & 2 \\
$16-35$ años & 4 & 7 & 11 \\
$36-60$ años & 2 & 10 & 12 \\
$>60$ años & 0 & 1 & 1 \\
\hline Total & 7 & 19 & 26 \\
\hline
\end{tabular}

Tabla 3. Localizaciones corporales de los aislamientos de $E$. floccosum de según sexo

\begin{tabular}{lccc}
\hline Localización & Mujeres & Hombres & Total \\
Plantar & 2 & 10 & 12 \\
Uñas pies & 1 & 8 & 9 \\
Tiña corporis & 3 & 0 & 3 \\
Inguinal & 1 & 1 & 2 \\
Total & 7 & 19 & 26 \\
\hline
\end{tabular}

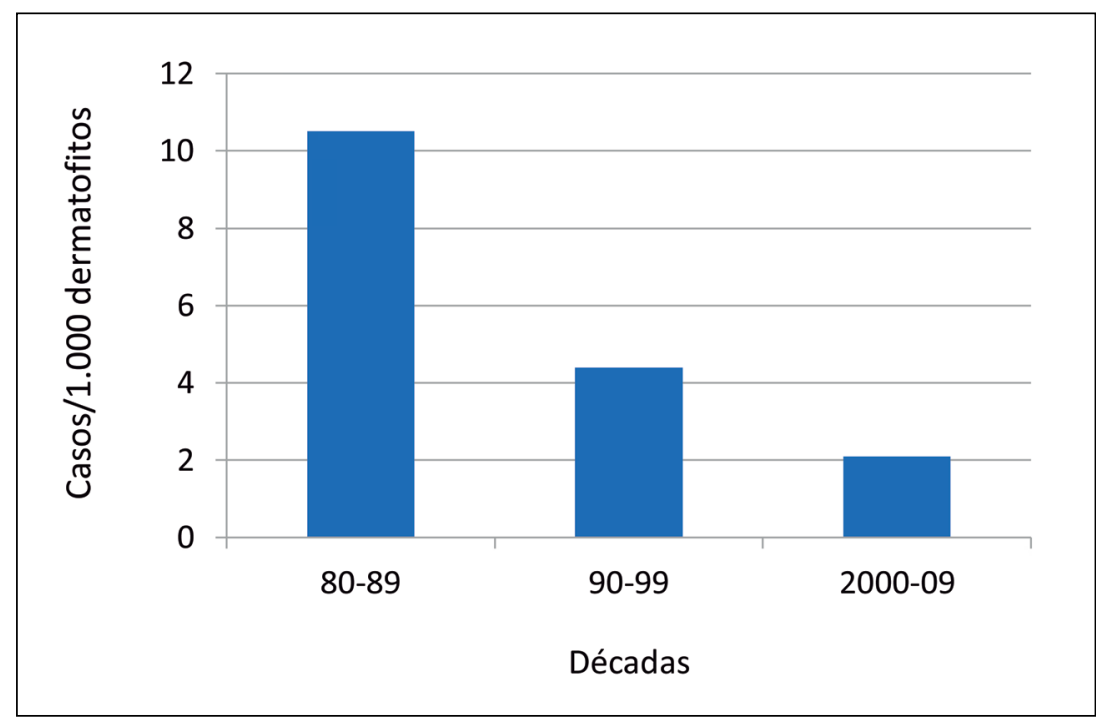

Gráfico 1. Tasa de aislamiento de E. floccosum (casos/1.000 dermatofitos) por década.
La tasa de aislamiento de E. floccosum en relación al resto de los dermatofitos disminuyó progresivamente desde la década de los 80 (10,5 casos por cada 1.000 dermatofitos) hasta la de 2000 (2,1 casos por cada 1.000 dermatofitos) (Figura 2). Lo mismo ocurrió al hacer la relación con el total de las muestras por cada década.

Los grupos etarios más afectados por este agente fueron el de 36-60 años, seguido por el de 16-35 y casi no hubo casos en los extremos de la vida (Tabla 2).

Las localizaciones más frecuentemente afectadas fueron la planta y uñas de los pies, tanto en hombres como mujeres (Tabla 3).

\section{Discusión}

Las dermatofitosis son infecciones frecuentes y se manifiestan como causas importantes de pérdida de cabello y uñas, inflamación, pústulas, picazón y descamación, por lo que son un desafiante problema en dermatología ${ }^{5}$. La corroboración de la sospecha diagnóstica mediante estudio micológico es fundamental para el médico clínico, por lo que debe ser solicitado antes del inicio del tratamiento ${ }^{3}$.

Epidemophyton floccosum, junto a las especies de los géneros Microsporum y Trichophyton son los agentes de las distintas formas clínicas de dermatofitosis ${ }^{3}$.

En el presente trabajo, las infecciones por E. floccosum predominaron en hombres, lo que es distinto a otras publicaciones donde, tanto en dermatomicosis como dermatofitosis, se describe mayor número de mujeres o ninguna diferencia por sexo y agente ${ }^{10-13}$. En cuanto a la edad de la población hay coincidencia entre esta y otras publicaciones debido a que las infecciones ocurrieron principalmente en grupos entre 30 y 60 años ${ }^{10-13}$.

Cambios significativos en la epidemiología, etiología y patrón clínico de estas infecciones se han observado en los últimos años en distintos continentes ${ }^{6,15}$. Las migraciones, la aparición de nuevos agentes antifúngicos, la popularización de los zapatos de cuero, zapatillas de deporte y la mejora de la higiene son probables responsables de este cambio $^{6,15}$. Zhan y cols., describieron distintos cambios epidemiológicos ocurridos durante los últimos 30 años en distintos países y continentes, donde T. rubrum sigue predominando en tiñas de piel y de uñas en todo el mundo, seguido por T. mentagrophytes, T. tonsurans y M. canis. Trichophyton schoenleinii, T. soudanense y M. audouinii siguen siendo frecuentes en África ${ }^{6}$.

En Chile, al igual que en otros países, T. rubrum, especie antropofilica, ha predominado ampliamente, seguido por T. mentagrophytes y M. canis $^{10}$. Epidemophyton floccosum es una especie de baja frecuencia en la mayoría de los países, incluso ha desaparecido en algunos $^{6,16}$. En este trabajo se muestra que esta especie no sólo es infrecuente en nuestro medio, sino que además ha 
disminuido progresivamente su frecuencia de aislamiento en las tres décadas estudiadas, tanto al compararlo con los otros géneros, como en relación al total de las muestras obtenidas por décadas. Esta situación es distinta a lo que ocurre en países asiáticos como Irán, donde es la segunda frecuencia luego de T. interdigitale ${ }^{16}$.

En nuestro trabajo, las infecciones por E. floccosum ocurrieron principalmente en adultos jóvenes y casi no hubo casos en las edades extremas de la vida, situación distinta a lo que ocurre con otros dermatofitos, por ejemplo, M. canis, que en los últimos años ha aumentado entre la población pediátrica y adulta mayor ${ }^{8,17}$.

Si bien se describe que E. floccosum puede afectar las distintas localizaciones de piel y anexos, principalmente se ha asociado a tiña de región inguinal, la cual puede comprometer genitales, región pubiana y piel de las zonas perineal y perianal ${ }^{1-3}$. Esto no coincide con nuestro trabajo donde sólo un paciente presentó lesión inguinal, siendo la región plantar la más afectada.

Epidemophyton floccosum es una especie antropofílica que ha disminuido su frecuencia en relación a las otras especies de dermatofitos, además de cambiar las localizaciones que tradicionalmente se describían como "típicas". Todo esto y dado los continuos cambios demográficos, higiénicos, migraciones, las condiciones de calidad de vida de las poblaciones, estimula a seguir vigilando en los decenios siguientes los probables cambios de ésta y otras especies de importancia clínica.

\section{Resumen}

Introducción: Las dermatofitosis son infecciones comunes en humanos, provocadas por hongos de los géneros Trichophyton, Microsporum y Epidermophyton. Objetivo: Determinar la frecuencia de E. floccosum en dermatofitos aislados en un laboratorio de la Región de Valparaíso durante las últimas tres décadas. Conocer las localizaciones más frecuentes, los grupos etarios y sexo de los pacientes afectados por este agente. Materiales y Método: Se revisaron los informes de cultivos superficiales con desarrollo de E. floccosum, Microsporum spp y Trichophyton spp de las tres últimas décadas del laboratorio de Micología de la Universidad de Valparaíso. Se registró en una planilla Excel el resultado del cultivo, edad, sexo y ubicación de la lesión de los pacientes. Resultados: El total de dermatofitosis con agente identificado fue de 6.780. En 26 casos hubo desarrollo de E. floccosum, de éstos, 73\% (19/26) fueron en hombres y con una edad promedio de 37 años. La frecuencia disminuyó progresivamente desde la década de los ochenta hasta la del 2000. El grupo etario más afectados fue el de 36-60 años. Las localizaciones más frecuentemente afectadas fueron la planta y uñas de los pies. Conclusiones: Existe una disminución progresiva de la frecuencia de aislamiento de E. floccosum en el período estudiado. El sexo masculino y el grupo etario de 36-60 años fueron los más afectados. Las localizaciones más frecuentes fueron la planta y uñas de los pies.

\section{Referencias bibliográficas}

1.- Arenas R. Dermatofitosis. Arenas R, editor. Micología Médica Ilustrada, $1^{\text {a }}$ ed. Ciudad de México: Editorial McGraw-Hill; 2004, p. 6194.

2.- Velasco M, García-Melgares L, Gimeno E, Roche E, Vilata J J. Dermatofitosis. Vilata J J, editor. Micosis Cutaneas, $1^{\text {a }}$ ed. Madrid: Editorial Médica Panamericana 2006; p. 49-72.

3.- Quindos G, Crespo V, Bonifaz A, Arenas R, Pereiro M, Giusiano G, et al. Micosis superficiales y subcutáneas. Quindos G, editor. Micología clínica, $1^{a}$ ed. Barcelona: Elsevier España 2015; p. 55-71.

4.- Molina de Diego A. Aspectos clínicos, diagnósticos y terapéuticos de las dermatofitosis. Enferm Infecc Microbiol Clin 2011; 29 (3): 33-9. file://C:/Users/pepe/ Downloads/S0213005X11700258_S300_ es.pdf.

5.- Fuller L C. Changing face of tinea capitis in Europe. Curr Opin Infect Dis. 2009; 22: 1158. ís: Sidoi: 10.1097/QCO.0b013e3283293d9b.

6.- Zhan P, Liu W. The changing face of dermatophytic infections worldwide. Mycopathologia 2017; 182 (1-2): 77-86. doi: 10.1007/s11046-016-0082-8.

7.- $\quad$ Kim S L, Lee K C, Jang Y H, Lee S J, Kim D W, Lee W J, et al. The epidemiology of dermatophyte infection in Southeastern Korea (1979-2013). Ann Dermatol. 2016; 28 (4): 524-7. doi: 10.5021/ad.2016.28.4.524.

8.- Cruz R, Carvajal L, Pérez S, Rodríguez V. Aislamiento de Microsporum spp. en dermatofitosis en pacientes de la región de Valparaíso. Chile. Rev Argent Dermatol 2017; 98 (1): 2-5. http://www.scielo.org.ar/ scielo.php?script $=$ sci_arttext\&pid $=\mathrm{S} 1851$ 300X2017000100005.

9.- Díaz M, Fich F, Salamanca L. Agentes etiológicos de micosis superficiales en un área de Santiago. Chile (1977-1987). Bol Micol 1990; 5: 5-8.

10.- Cruz R, Ponce E, Calderón L, Delgado N, Vieille P, Piontelli E. Micosis superficiales en la ciudad de Valparaíso, Chile: Período $2007-$ 2009. Rev Chilena Infectol 2011; 28 (5): 404-9. DOI: 10.4067/S0716-10182011000600002.

11.- Piontelli E, Toro M, Casanova D, Jara D. Micosis superficiales en pacientes de servicios dermatológicos de la V Región: Estudios de prevalencia en el período 1984-1989. Bol Micol 1991; 6: 63-8.

12.- Díaz M, Díaz P, Rodríguez J, Muñoz A.
Evaluación del perfil de sensibilidad in vitro de aislamientos clínicos de Trichophyton mentagrophytes y Trichophyton rubrum en Santiago, Chile. Rev Iberoam Micol 2015; 32 (2): 83-7. DOI: 10.1016/j.riam.2013.12.002.

13.- Díaz M, Roessler P, Fich F, Gómez O, Ostornol P, Pérez L. Dermatofitosis. Etiología y susceptibilidad en tres centros hospitalarios de Santiago (Chile). Bol Micol 2002; 17: 101-07.

14.- Zaror L, Bustamante X. Viabilidad de dermatofitos en muestras clínicas almacenadas. Bol Micol 1998; 13 (1-2): 23-7.

15.- Havlickova B, Czaika V A, Friedrich M. Epidemiological trends in skin mycoses worldwide. Mycoses. 2008; 51: 2-15. doi: 10.1111/j.1439-0507.2008.01606.x.

16.- Rezaei-Matehkolaei A, Rafiei A, Makimura K, Graser Y, Gharghani M, Sadeghi-Nejad B. Epidemiological aspects of dermatophytosis in Khuzestan, southwestern Iran, an update. Mycopathologia 2016;181: 547-53.

17.- Miklic P, Skerlev M, Budimcic D, Lipozencic J. The frequency of superficial mycoses according to agents isolated during a ten-year period (1999-2008) in Zagreb area, Croatia. Acta Dermatovenerol Croat 2010; 18 : 92-8. 